\title{
Instituting robotic pediatric urologic surgery in the Canadian healthcare system: Evaluating the feasibility and outcomes of robot-assisted pyeloplasty and ureteric reimplantation
}

Noah Stern; Peter Wang; Sumit Dave

London Health Sciences Centre, Western University, London, ON, Canada

Cite as: Stern N, Wang P, Dave S. Instituting robotic pediatric urologic surgery in the Canadian healthcare system: Evaluating the feasibility and outcomes of robot-assisted pyeloplasty and ureteric reimplantation. Can Urol Assoc J 2020 September 28; Epub ahead of print. http://dx.doi.org/10.5489/cuaj.6604

Published online September 28, 2020

$* * *$

\section{Abstract}

Introduction: Robotic pediatric urologic surgery has gained widespread adoption over the last decade. This article describes our experience in instituting the first pediatric urologic robotic surgery program in Canada. We evaluated the feasibility and safety of instituting pediatric robotassisted urologic surgery and report our early outcomes for robot-assisted pyeloplasty (RAP) and ureteric reimplantation (RUR).

Methods: We prospectively evaluated all patients undergoing RAP and RUR by a single surgeon from June 2013 to March 2019. Demographic and clinical data were prospectively collected and included sex, age, and preoperative grade of hydronephrosis or reflux. Descriptive statistics were performed, and comparisons were made using Student's t-tests where appropriate. Success was defined as resolution or significant improvement of hydronephrosis following RAP and absence of recurrent urinary tract infection (UTI) and/or persistent vesicoureteric reflux (VUR) following RUR. Complications were described using the Clavien-Dindo system.

Results: A total of 52 RAPs and 24 RURs were performed with a minimum of six months followup. Forty-five RAP patients met criteria for success, while diagnostic imaging of success in the form of MAG-3 Lasix renograms was documented in the remaining seven for an overall success of $100 \%$. Sixteen RUR patients met criteria for success and seven showed resolution of VUR on imaging following their first UTI, for an overall success rate of $96 \%$. Operative times progressively improved from $204 \pm 35$ minutes to $121 \pm 15$ minutes in the RAP group and from $224 \pm 52$ to $132 \pm 39$ minutes in the RUR group. In the RAP cohort, one Clavien grade II and four Clavien grade III complications were noted, while three Clavien grade III complications were 
noted in the RUR cohort.

Conclusions: Despite limited case volumes, robotic pediatric urologic surgery can be integrated into the Canadian healthcare system with success rates comparable to reported literature. However, compared to open surgery, RAP and especially RUR warrant further study to ensure lack of significant complications noted in our study.

\section{Introduction}

Since first being demonstrated to be safe and feasible in the pediatric urologic population in the early 2000s, robot-assisted surgery has established itself as a viable and sometimes preferred approach for surgical management of common pediatric urologic conditions. ${ }^{1-3}$ The purported patient benefits of shorter lengths of stay, decreased analgesia requirements, improved cosmesis coupled with the surgeon benefits of superior 3-dimentional visualization, ergonomics, and range of motion have driven an increase in robotic utilization. ${ }^{2,4,5}$ Currently, over $40 \%$ of all pediatric pyeloplasties performed in the United States use a robot-assisted approach. ${ }^{2-5}$

As the index robot-assisted pediatric procedure, robot-assisted pyeloplasty (RAP) has the largest body of evidence with several multi-institutional studies showing outcomes equivalent or superior to open and laparoscopic approaches with shorter hospital stays and decreased complication rates. ${ }^{6-9}$ The use of robot assistance for extra-vesical ureteral reimplantation (RUR), however, is more controversial with mixed results. ${ }^{10-12}$

In 2013, the pediatric urology program at Western University established a pediatric robotic urology program and studied the process prospectively over 5 years to assess the feasibility and safety of robot-assisted pediatric urologic surgery in our Canadian health care system. This prospective cohort study describes the results of a consecutive series of patients undergoing RAP and RUR performed by a single surgeon.

\section{Methods}

This prospective cohort study includes all consecutive RAP and RUR performed at our institution between June 2013 and March 2019, with a minimum 6-months follow up period to assess feasibility, success, and complications following RAP and RUR.

\section{Establishing the program}

The pediatric hospital at our institution is situated within our adult hospital providing a unique opportunity to establish a pediatric robotic program without the additional financial burden of purchasing an independent robotic unit. The primary surgeon and the lead nurse visited the pediatric urology robotic program at the Children's Hospital of Philadelphia and a surgeon from that program mentored and was present during the initial 4 cases. A dedicated team of nurses trained in robotic surgery was formed to assist during subsequent cases. A prospective pre- 
defined database of all patients undergoing pediatric robotic surgery was created and the study approved by our Institutional Review Board.

\section{Patient selection}

This cohort included all pediatric patients between 6 months and 18 years of age undergoing RAP and RUR. Patients were selected through shared decision making between the surgeon, patient, and parents. RAP was offered to patients older than 6 months of age who presented with antenatally diagnosed persistent hydronephrosis and evidence of ureteropelvic junction obstruction (UPJO) or delayed presentation secondary to Dietls' crisis based on ultrasonographic imaging and mercapto-acetyl-triglycine (MAG-3) Lasix renogram. Similarly, RUR was offered to children over 6 months of age who required surgical intervention for $\geq$ grade III vesicoureteric reflux (VUR) associated with breakthrough recurrent urinary tract infections (UTIs) with or without renal scarring on dimercaptosuccinic acid (DMSA) scans. The indications for surgical intervention for both RAP and RUR were identical to those for open and laparoscopic pyeloplasty and ureteric reimplantation.

\section{Surgical technique}

All surgeries were performed using the da Vinci Si Surgical System ${ }^{\circledR}$ using a 3-port technique, without an assistant port. An $8 \mathrm{~mm}$ camera port was placed using an open Hasson technique through the umbilicus and two $5 \mathrm{~mm}$ robotic instrument ports were placed as required. All procedures utilized the following robotic instruments: Maryland forceps, needle driver, monopolar hook and scissor. Resident involvement, primarily during intracorporal suturing, was gradually increased throughout the study as surgeon experience grew.

\section{Robot-assisted pyeloplasty}

A 2-way Foley catheter with Methylene blue saline access was placed prior to patient positioning to allow assessment of the anastomosis competence and antegrade ureteric stent placement. An Anderson-Hynes dismembered pyeloplasty technique was performed for all patients using an intraperitoneal trans-mesenteric approach. The ureter was transposed anterior to the crossing vessel when indicated. A percutaneous hitch stitch on the renal pelvis was utilized to aid in dissection and suturing. Uretero-pelvic anastomosis was performed using 5-0 PDS in a continuous manner between the renal pelvis and the spatulated ureter. An antegrade double $\mathrm{J}$ ureteric stent was placed across the anastomosis and a Blake perforated drain was placed for drainage. The stent was subsequently removed 4-6 weeks later under general anesthetic.

\section{Robot-assisted ureteric reimplantation}

All patients underwent an extravesical ureteric reimplantation using a Lich-Gregoir technique. Patients were position in Trendelenburg position after port placement. The peritoneum distal to the broad ligament was incised to expose the ureters posterior to the bladder. The ureters were 
dissected adequately preserving their adventitial blood supply. A percutaneous hitch stitch was placed in the bladder to elevate the posterior bladder wall and the bladder was distended with $25 \mathrm{~mL}$ normal saline. A detrusor tunnel was created using a 4:1 ratio of ureteric diameter to tunnel length. The ureters were then re-implanted without stents by closing the detrusor muscle tunnel with interrupted 5-0 PDS sutures, incorporating the ureteral serosa in a few bites.

\section{Surgical timing}

Both RAP and RUR where sectioned into discrete steps, capturing a total time from port insertion to port removal. This time did not capture the 10-20 minutes for each procedure which included an exact skin to skin timing. Timing of robot set up and dismantling are not included.

\section{Case costing}

Costing for disposables and robotic instruments per use were calculated for both RAP and RUR using the final 5 cases for each procedure. This costing does not include fixed robot costs, yearly maintenance contracts, or staffing. All material used was recorded and catalogued with costs determined by standard internal procedure and catalogue.

\section{Outcomes}

The primary outcome for this study was surgical success within a follow-up period of 6 months and identical to standards of assessment applied for open pyeloplasty and ureteric reimplantation. For patients undergoing RAP, success was defined as postoperative resolution or significant decrease in the grade of hydronephrosis and antero-posterior (AP) renal pelvic diameter on ultrasound imaging and/or resolution of symptoms. If patients did not exhibit a significant decrease in hydronephrosis a MAG-3 renogram was performed and decreased T-half times and/or preserved or improved differential renal function and improved drainage curves were utilized to assess postoperative success. For patients undergoing RUR success was defined as resolution of UTIs without continuous antibiotic prophylaxis. Postoperatively, if patients had a culture proven febrile UTI a VCUG was performed to rule out persistent VUR. Given this was a new program, the threshold to perform a MAG-3 renogram or a VCUG was subjectively lower in this cohort compared to open surgical procedures.

\section{Data and statistical analysis}

Patient demographic data, preoperative, intraoperative and postoperative details were collected prospectively. Outcomes were analyzed using Student's t-test using SPSS 25. Complications were assessed using the Clavien-Dindo classification system. ${ }^{13}$ 


\section{Results}

\section{RAP}

52 RAP were performed in 35 boys and 17 girls at a mean age of 87 months (range 8-222). Table 1 shows detailed patient demographics. The mean robotic operative time, defined as time from start of port insertion to robot undocking was $157 \pm 39$ minutes and progressively decreased from $204 \pm 35$ minutes in the first quartile to $121 \pm 15$ minutes in the last quartile (Figure $1, \mathrm{p}<0.0001$ ). Resident console time increased from $12 \pm 11$ minutes to $21 \pm 10$ minutes between the first and final quartile. All procedures were performed robotically with no conversions to open surgery. Average RAP case was costed at $\$ 2550$. No major intraoperative complications were noted. Four early postoperative complications were observed: one Clavien grade II and three Clavien grade III (Table 2). The mean length of hospital stay was 3.5 days (range 2-11).

The mean follow-up post RAP was $25 \pm 17$ months. In patients with at least 6 months of follow up, 45/52 patients showed significant improvement in their degree of hydronephrosis, with an average reduction of SFU grade of hydronephrosis of 1-2 grades. A further 5 patients with residual hydronephrosis underwent repeat MAG-3 renograms showing interval improvement in t-half times and drainage curves with preserved or improved differential function. Two patients with improved but persistent hydronephrosis initially presented with Dietls' crisis. These patients were asymptomatic post-operatively and opted to defer further investigation.

\section{RUR}

Robot assisted ureteric reimplantation was performed for 1 boy and 23 girls at a mean age of 65 months (range 22-148), presenting with unilateral (8 patients) or bilateral (16 patients) VUR. Table 1 shows detailed patient demographics. The mean operative time for RUR, defined as time from start of port insertion to port removal was $175 \pm 50$ minutes and progressively decreased from $224 \pm 52$ in the first quartile minutes to $132 \pm 39$ minutes in the last quartile (Figure 1). All procedures were performed robotically with no conversions to open surgery. A sample RUR case was costed at $\$ 2600$. No major intraoperative complications or robotic technical issues were noted. Four mucosal breaches were identified during dissection of the mucosal tunnel and repaired appropriately. Three early post operative Clavien grade III complications were noted (Table 2). The mean length of hospital stay was 4 days (range 2-20).

The mean follow-up for patients undergoing RUR was $34 \pm 17$ months. A total of 16 patients showed post-operative resolution of their UTIs without continuous antibiotic prophylaxis. In 8 patients with recurring UTIs following RUR a VCUG was performed where 7 showed resolution of reflux and one downgraded their VUR grade from 4 to 2. One patient developed contralateral reflux with febrile UTIs subsequently requiring reimplantation of the contralateral ureter. 


\section{Discussion}

Open and laparoscopic surgery remain the standard of care in the treatment of pediatric VUR and UPJO, with success rates up to $95 \%$ for open ureteric reimplantation and $82-100 \%$ for open and laparoscopic pyeloplasty. ${ }^{22-24}$ Although there is a plethora of literature on the outcomes of robotic pediatric urology in the USA, a Canadian experience with pediatric robotic surgery is currently lacking. This prospective study, a Canadian first, examined a pediatric urologic series of patients undergoing RAP and RUR. Surgical success in our series was $100 \%$ in the RAP cohort and $96 \%$ in the RUR cohort.

In pediatric patients undergoing RAP, multiple multi-institution studies have shown success rates comparable to open and laparoscopic surgery, ranging between $85-100 \% .{ }^{8,25,26}$ In our series all patients underwent successful pyeloplasties, with none requiring redo-procedures. However, 4 (7.7\%) of these patients required an additional procedure secondary to a complication.

The advantage of minimally invasive surgery come at the expense of increased operative times. National American median operative times cite open pyeloplasty to be nearly $40 \%$ faster than robotic and $25 \%$ faster than laparoscopic approaches, however high volume centres can see operative times significantly lower than stated medians. ${ }^{8,22,27}$ This series showed a $41 \%$ decrease in operative time from a mean of 223 minutes in the first quartile to 131 minutes in the final quartile, despite increasing resident involvement. Our operative time does not include robot preparation or dismantling time which makes direct comparison to previously published data difficult. Regardless, it is evident that despite lower case volumes per year (9 per year), one can overcome the learning curve associated with RAP and demonstrate significantly decreased operative times while maintaining comparable success rates to open/laparoscopic procedures. While the first 15 cases were performed solely by the lead surgeon, resident console time primarily in the form of intracorporeal suturing - progressively increased throughout the series as primary surgeon proficiency grew, ensuring limited impact on the trainee exposure. Laparoscopic pyeloplasty is a well-established procedure in Canada with several series reported. ${ }^{29-32}$ Studies investigating the safety and learning curve adopting a minimally invasive technique have consistently found progressive improvements in operative times and similar success rates to the open approach, though costs are approximately $\$ 1500$ more per robotic case. ${ }^{30,31,33}$ Meta-analyses comparing RAP to open or laparoscopic pyeloplasty have shown equivalent outcomes with the potential for decreased lengths of stay and analgesic requirements for RAP and a complication rate of $4-11 \%{ }^{8,34}$ These benefits come at the cost of longer operative times and cost per case..$^{23,35}$

The high degree of technical complexity required for laparoscopic ureteric reimplantation explains the fact that less than $2 \%$ of all pediatric extravesical ureteric reimplantations are performed using a minimally invasive technique. ${ }^{10}$ Therefore, RUR is an innovative step utilizing robotic assistance to perform ureteric reimplantation using a minimally invasive 
approach. Multiple centres have demonstrated the feasibility of RUR, however its success and safety compared to open surgery is yet to be proven. Retrospective case series and meta-analyses have shown that RUR is associated with a range of success rates (73-99.7\%) and possible higher complication rates (0-30\%) compared to open reimplantation. ${ }^{7,11,12,14,15,36-38}$

In our series $23 / 24$ patients met the criteria for success and one required subsequent open reimplantation. The RUR cohort showed a higher than expected complication rate at $12 \%$. It is debatable whether these complications are inherent to RUR or whether they reflect the learning curve associated with a new surgical approach, as all complications occurred in the first half of this cohort. Analyzing the complications, modifiable system errors were recognized and addressed in order to prevent recurrence. Initially, the use pediatric robotic instruments for RUR limited access to bipolar forceps, which is only available as an 8-mm adult instrument. Reviewing the surgical video, two instances of the use of monopolar cautery to control minimal ureteral bleeding was noted, which subsequently resulted in ureteric leaks. We are now using adult robotic instruments for RUR specifically to have access to a bipolar instrument to decrease the risk of thermal ureteric injury.

\section{Surgical costs}

The initiation of a robotics program requires a hefty initial investment and endorsement from the institution, the department, the surgeon, and the patient. We were uniquely situated to explore the role of robotic surgery in the pediatric urologic population in Canada as our pediatric hospital is physically located with our adult hospital and shares operating rooms. This allowed for cost sharing of the robotic system and avoided the requirements to purchase an independent pediatric unit besides allowing for a single cohort of dedicated and trained nursing staff.

Unfortunately, given the nature of the Canadian compared to the American system direct cost comparison is difficult. A sample pediatric RAP in our cohort was costed at \$2550 CAD, while a sample RUR cost $\$ 2600$. These costs do not account for any non-operative costs incurred during the hospital stay, the purchase and maintenance of the robot or any post operative visits, investigations, and procedures (including stent removal). Bennet Jr et al. used administrative data to query all pediatric pyeloplasties performed at 43 tertiary care pediatric hospitals in the United States with estimated costs ranging between \$10,160-17,418 USD (\$14,342-24,588 CAD) per case, with $72 \%$ of the costs relating to operating room, anesthesia and operative supplies. ${ }^{39}$ These charges were calculated accounting for both the amount charged by hospitals and the amount actually collected. While no explicit charge for the purchase or maintenance of the robot is listed it is likely incorporated into this fee. While direct comparison is difficult, it does appear that the robotic costs in the Canadian system may be more affordable than anticipated. Further direct and complete cost comparison with open or laparoscopic surgery is required to further study the true costs of robotic surgery in Canada. Efforts to further decrease 
costs could involve the use of a external/internal Salle stent as opposed to a double J stent, with an estimated cost saving $\$ 565$ and one less general ansesthetic. ${ }^{40}$

In our institution it is standard practice to admit patients for 2-3 days regardless of technique used, though arguments can be made for earlier discharge for those undergoing RAP. Majority of our patients did meet discharge criteria earlier than their eventual discharge but we chose to be overly cautious during this initial robotic experience. This has likely inflated our hospital stays for both RAP and RUR. Given our initial experience, more aggressive discharging practices have been instituted. In patients with extended stays, the majority were patients experiencing post operative complications including urine leaks and postoperative infections.

\section{Limitations}

This prospective study has limitations worth considering. We defined success using open surgery criteria to minimize unnecessary investigation in pediatric patients. This resulted in some patients not undergoing postoperative MAG-3 renograms or VCUGs. This was consistent with the standard post-operative protocol as used at our institution following open surgery and since these robotic procedures replicated steps of open operations, they have been previously deemed those protocols as appropriate. ${ }^{34,41}$ In addition, our threshold to perform these tests was subjectively lower in this robotic case series. Furthermore, the lack of a comparable group of matched patients undergoing laparoscopic or open pyeloplasty and ureteric reimplantation limits the ability to definitively evaluate the robotic approach at our centre. Finally, the operative timing chosen from port insertion to port removal was chosen as it represents the time most under the surgeon's control, independent from external and systemic factors. An addition of 1020 minute per case is likely necessary for comparison to previously published series that include total OR time.

\section{Conclusions}

Pediatric robotic urologic surgery can be adopted into the Canadian healthcare system yielding outcomes comparable to open surgery. In addition, despite low case volumes per year, outcomes and complication rates are comparable to high volume centres in the US. Operative times decrease significantly after 20 cases for both RAP and RUR. Concerns exist regarding a higher complication rate noted for both RAP and RUR and though this may reflect an early learning curve deficiency, further prospective studies are indicated to define this complication rate noted. 


\section{References}

1. Gettman MT, Neururer R., Bartsch G, et al. Anderson-Hynes dismembered pyeloplasty performed using the da Vinci robotic system. Urology 60, 509-513 (2002).

2. Varda, BK, Wang Y, Chung B, et al. Has the robot caught up? National trends in utilization, perioperative outcomes, and cost for open, laparoscopic, and robotic pediatric pyeloplasty in the United States from 2003 to 2015. Journal of Pediatric Urology 14, 336.e1-336.e8 (2018).

3. Cundy TP, Shetty K, Clark J, et al. The first decade of robotic surgery in children. Journal of Pediatric Surgery 48, 858-865 (2013).

4. Taktak S, Llewellyn O, Aboelsoud M, et al. Robot-assisted laparoscopic pyeloplasty versus laparoscopic pyeloplasty for pelvi-ureteric junction obstruction in the paediatric population: a systematic review and meta-analysis. Therapeutic Advances in Urology 11, 175628721983570 (2019).

5. Howe A, Kozel Z, Palmer L. Robotic surgery in pediatric urology. Asian Journal of Urology 4, 55-67 (2017).

6. Silay MS, Spiinoit AF, Undre S, et al. Global minimally invasive pyeloplasty study in children: Results from the Pediatric Urology Expert Group of the European Association of Urology Young Academic Urologists working party. Journal of Pediatric Urology 12, 229.e1-229.e7 (2016).

7. Yee DS, Shaberg AM, Duel BP, et al. Initial comparison of robotic-assisted laparoscopic versus open pyeloplasty in children. Urology 67, 599-602 (2006).

8. Minnillo BJ, Cruz J, Sayao R, et al. Long-term experience and outcomes of robotic assisted laparoscopic pyeloplasty in children and young adults. Journal of Urology 185, 1455-1460 (2011).

9. Bansal D, Cost N, DeFoor W, et al. Infant robotic pyeloplasty: comparison with an open cohort. Journal of Pediatric Urology 10, 380-385 (2014).

10. Bowen D, Maasse M, Liu D et al. Use of pediatric open, laparoscopic and robot-assisted laparoscopic ureteral reimplantation in the United States: 2000 to 2012. Journal of Urology 196, 207-212 (2016).

11. Akhavan A, Avery D, Lendvay T. Robot-assisted extravesical ureteral reimplantation: Outcomes and conclusions from 78 ureters. Journal of Pediatric Urology 10, 864-868 (2014).

12. Grimsby G, Dwyer M, Jacobs M, et al. Multi-institutional review of outcomes of robotassisted laparoscopic extravesical ureteral reimplantation. The Journal of Urology 193, 1791-1795 (2015).

13. Dindo D, Demartines N, Clavien, PA. Classification of surgical complications. Annals of Surgery 240, 205-213 (2004).

14. Marchini G, Hong Y, Minnillo Bet al. Robotic assisted laparoscopic ureteral reimplantation in children: case matched comparative study with open surgical approach. J. Urol. 185, 1870-1875 (2011). 
15. Lee RS, Retik A, Borer J, et al. Pediatric robot assisted laparoscopic dismembered pyeloplasty: comparison with a cohort of open surgery. Journal of Urology 175, 683-687 (2006).

16. Hubert K, Kokorowski P, Huang L, et al. Durability of anti-reflux effect of ureteral reimplantation for primary vesicoureteral reflux: findings on long-term cystography. Urology 79, 675-679 (2012).

17. Chertin B, Prosolovich K, Aharon S, et al. Surgical reimplantation for the correction of vesicoureteral reflux following failed endoscopic injection. Adv Urol 2011, (2011).

18. Maheshwari R, Ansari M, Mandhani A, et al. Laparoscopic pyeloplasty in pediatric patients: The SGPGI experience. Indian J Urol 26, 36-40 (2010).

19. Kavoussi L, Peters C. Laparoscopic pyeloplasty. J. Urol. 150, 1891-1894 (1993).

20. Ben Slama M, Salomon L, Hoznek A et al. Extraperitoneal laparoscopic repair of ureteropelvic junction obstruction: initial experience in 15 cases. Urology 56, 45-48 (2000).

21. Seo I, Oh T, Lee J. Long-term follow-up results of laparoscopic pyeloplasty. Korean J Urol 55, 656-659 (2014).

22. Jodal U, Smellie J, Lax H, et al. Ten-year results of randomized treatment of children with severe vesicoureteral reflux. Final report of the International Reflux Study in Children. Pediatr. Nephrol. 21, 785-792 (2006).

23. Cundy T, Harling L, Hughes-Hallett A, et al. Meta-analysis of robot-assisted vs conventional laparoscopic and open pyeloplasty in children. BJU International 114, 582594 (2014).

24. Traxel E, Minevich E, Noh P. A review: the application of minimally invasive surgery to pediatric urology: upper urinary tract procedures. Urology 76, 122-133 (2010).

25. Peters C. Robotically assisted surgery in pediatric urology. Urologic Clinics of North America 31, 743-752 (2004).

26. Hollis M, Cho P, Yu R. Pediatric robot-assisted laparoscopic pyeloplasty. Am J Robot Surg 2, 1-8 (2015).

27. Atug, F., Woods, M., Burgess, S. V., Castle, E. P. \& Thomas, R. Robotic assisted laparoscopic pyeloplasty in children. J. Urol. 174, 1440-1442 (2005).

28. Varda B Johnson E, Clark C, et al. National trends of perioperative outcomes and costs for open, laparoscopic and robotic pediatric pyeloplasty. Journal of Urology 191, 10901096 (2014).

29. Eassa W, Al Zahrani A, Jednak R, et al. A novel technique of stenting for laparoscopic pyeloplasty in children. Journal of Pediatric Urology 8, 77-82 (2012).

30. Braga, L. H. et al. Pediatric laparoscopic pyeloplasty in a referral center: lessons learned. J. Endourol. 21, 738-742 (2007).

31. Braga L, Lorenzo A, Madhi M, et al. Comparative analysis of 3 different approaches for Pediatric Pyeloplasty. Journal of Pediatric Urology 4, S17-S18 (2008).

32. Abou-Haidar H, Al-Qaoud T, Jednak R, et al. Laparoscopic pyeloplasty: Initial experience with 3D vision laparoscopy and articulating shears. Journal of Pediatric Urology 12, 426.e1-426.e5 (2016). 
33. Moore K, Lorenszo A, Tuner S et al. Prospective cost analysis of laparoscopic vs. open pyeloplasty in children: Single centre contemporary evaluation comparing two procedures over a 1-year period. Can Urol Assoc J 7, 94-98 (2013).

34. Riachy E, Cost N, Defoor W, et al. Pediatric standard and robot-assisted laparoscopic pyeloplasty: a comparative single institution study. Journal of Urology 189, 283-287 (2013).

35. Autorino R, Eden C, El-Ghoneimi A, et al. Robot-assisted and laparoscopic repair of ureteropelvic junction obstruction: a systematic review and meta-analysis. European Urology 65, 430-452 (2014).

36. Sávio L, Nguten H. Robot-assisted laparoscopic urological surgery in children. Nature Reviews Urology 10, 632-639 (2013).

37. Edwards A, Peters C. Managing vesicoureteral reflux in children: making sense of all the data. F1000Res 8, (2019).

38. Kasturi S, Sehgal S, Christman M, et al. Prospective long-term analysis of nerve sparing extravesical robotic-assisted laparoscopic ureteral reimplantation. Urology 79, 680-683 (2012).

39. Bennett W, Whittam B, Szymanski K, et al. Validated cost comparison of open vs. robotic pyeloplasty in American children's hospitals. J Robotic Surg 11, 201-206 (2017).

40. Braga L, Lorenzi A, Farhat W, et al. Outcome analysis and cost comparison between externalized pyeloureteral and standard stents in 470 consecutive open pyeloplasties. J. Urol. 180, 1693-1698; discussion1698-1699 (2008).

41. Gatti J, Amstutz S, Bowlin P, et al. Laparoscopic vs open pyeloplasty in children: results of a randomized, prospective, controlled trial. Journal of Urology 197, 792-797 (2017). 


\section{Figures and Tables}

Fig.1. Progressive decreasing operative times for robot assisted pyeloplasty (green) and ureteric reimplantation (blue).

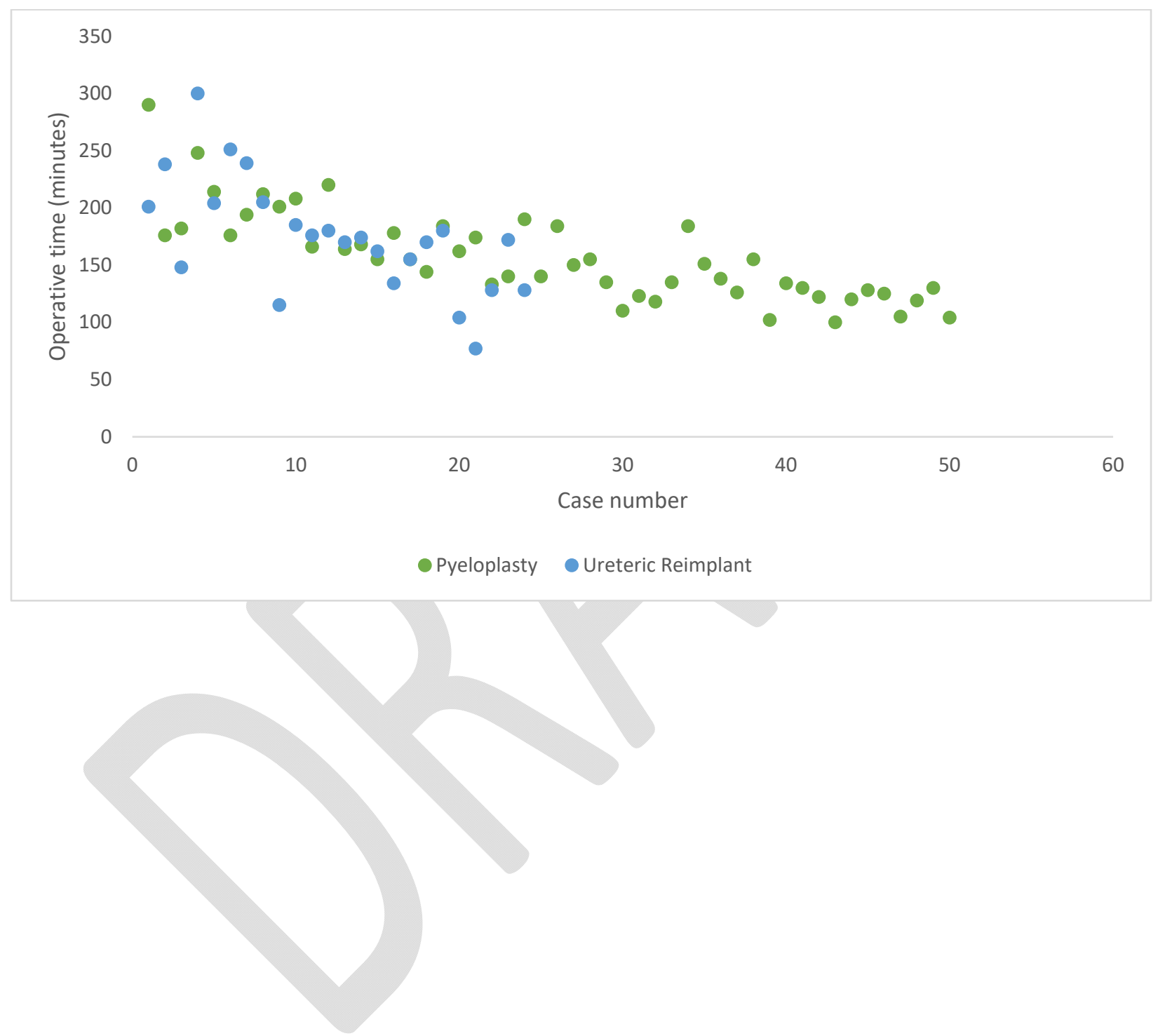




\begin{tabular}{|l|c|c|}
\hline \multicolumn{3}{|c|}{ Table 1. Demographics for patients undergoing RAP and RUR } \\
\hline & RAP & RUR \\
\hline Number of patients & 52 & 24 \\
\hline Median age (range) & $87(8-222)$ & $68(22-148)$ \\
\hline Gender & & 1 \\
\hline Male & 17 & 23 \\
\hline Female & & \\
\hline Presenting complaint(s) & 24 & \\
\hline Hydronephrosis & 14 & \\
\hline Pain & 4 & 17 \\
\hline UTI & & 14 \\
\hline Renal scarring & 10 & 1 \\
\hline Other & & 3 \\
\hline Laterality & 34 & 6 \\
\hline Left & 18 & 17 \\
\hline Right & & \\
\hline Bilateral & & \\
\hline Degree of hydronephrosis & 2 & \\
\hline I & 3 & \\
\hline II & 22 & 11 \\
\hline III & 25 & \\
\hline IV & & \\
\hline Degree of VUR & & \\
\hline I & & \\
\hline II-III & & \\
\hline IV-V & & \\
\hline & & \\
\hline
\end{tabular}

RAP: robot-assisted pyeloplasty; RUR: robot-assisted ureteric reimplantation; UTI: urinary tract infection; VUR: vesicoureteric reflux. 


\begin{tabular}{|c|c|c|c|}
\hline & Complication & Treatment & Outcome \\
\hline \multicolumn{4}{|l|}{ RAP } \\
\hline $\begin{array}{l}\text { Clavien grade } \\
\text { II }\end{array}$ & UTI & Intravenous antibiotics & Complete resolution \\
\hline \multirow[t]{4}{*}{$\begin{array}{l}\text { Clavien grade } \\
\text { III (b) }\end{array}$} & $\begin{array}{l}\text { Stent in ureter proximal } \\
\text { to uretero-vesical } \\
\text { junction }\end{array}$ & Antegrade stent retrieval & Complete resolution \\
\hline & Urine leak & Nephrostomy tube & Complete resolution \\
\hline & Urine leak & Nephrostomy tube & Mild hydronephrosis \\
\hline & $\begin{array}{l}\text { Unrecognized jejunal } \\
\text { enterotomy }\end{array}$ & $\begin{array}{c}\text { Exploratory laparotomy, } \\
\text { primary repair }\end{array}$ & Complete resolution \\
\hline \multicolumn{4}{|l|}{ RUR } \\
\hline \multirow{3}{*}{$\begin{array}{l}\text { Clavien grade } \\
\text { III }(\mathrm{a} / \mathrm{b})\end{array}$} & Suspected bladder leak & Foley catheter & Complete resolution \\
\hline & Ureteric injury & Ureteric stent placement & Complete resolution \\
\hline & Ureteric injury & Ureteric stent placement & Complete resolution \\
\hline
\end{tabular}

RAP: robot-assisted pyeloplasty; RUR: robot-assisted ureteric reimplantation; UTI: urinary tract infection. 